Check for updates

Cite this: Phys. Chem. Chem. Phys. 2021, 23, 8937

DOI: $10.1039 / d 1 c p 90063 c$

rsc.li/pccp

\section{Correction: Multifaceted aspects of charge transfer}

\author{
James B. Derr, (D) a Jesse Tamayo, (D) ${ }^{b}$ John A. Clark, (D) Maryann Morales, (D) ${ }^{b}$ \\ Maximillian F. Mayther, (D) ${ }^{\mathrm{b}}$ Eli M. Espinoza, (D) ${ }^{\mathrm{b}}$ Katarzyna Rybicka-Jasińska (D) ${ }^{\mathrm{c}}$ and \\ Valentine I. Vullev (D) *abcd
}

Correction for 'Multifaceted aspects of charge transfer' by James B. Derr et al., Phys. Chem. Chem. Phys., 2020, 22, 21583-21629, DOI: 10.1039/d0cp01556c.

The published version of this manuscript included errors in Fig. 6c, in which the radical cation should have been on 'B' not ' $\mathrm{D}$ ', and throughout the paragraph beginning 'The polyprolines do not have hydrogen-bonding...' on page 21597. The correct figure and text for the paragraph are shown below.

The polyprolines do not have hydrogen-bonding networks because they contain only tertiary amides along their backbones. Isomerization of the peptide bonds in polyprolines between $E$ and $Z$ alters the type of helical conformation and changes the magnitude and the direction of the macrodipole. Specifically, comprising peptide bonds in their $Z$-conformations, polyproline type I (PPI) has a dipole of $4.1 \mathrm{D}$ per residue that points from its $\mathrm{N}$ - to its C-terminus, which is opposite to $\alpha$ - and $3_{10}$-helices containing all $Z$-amides. ${ }^{231,235}$ Conversely, polyproline type II (PPII) contains $E$-amides and has a dipole of $1.5 \mathrm{D}$ per residue, pointing from its $\mathrm{C}$ - to its $\mathrm{N}$-terminus. ${ }^{231,235}$ Because of this difference in the magnitude of the macrodipole, changes in solvent polarity induce transitions between PPI and PPII conformations ${ }^{236}$ which can serve as an electret switch.

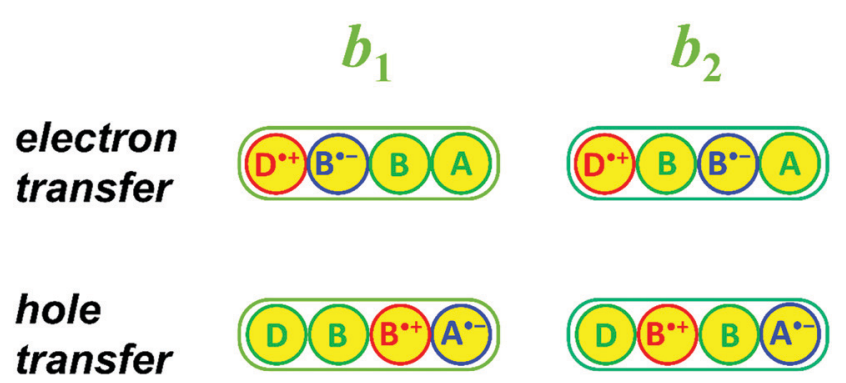

Fig. 6 (c) Charge distribution in the bridging states, $b_{1}$ and $b_{2}$, where the transferred electron is on the LUMOs, or the transferred hole is on the HOMOs, of the bridging units.

The Royal Society of Chemistry apologises for these errors and any consequent inconvenience to authors and readers.

\footnotetext{
${ }^{a}$ Department of Biochemistry, University of California, Riverside, CA 92521, USA. E-mail: vullev@ucr.edu

${ }^{b}$ Department of Chemistry, University of California, Riverside, CA 92521, USA

${ }^{c}$ Department of Bioengineering, University of California, Riverside, CA 92521, USA

${ }^{d}$ Materials Science and Engineering Program, University of California, Riverside, CA 92521, USA
} 